\title{
A comparison of FOLFIRI (Folinic Acid, Fluorouracil and Irinotecan) Plus Bevacizumab and FLOLFIRI Plus Aflibercept as Second-Line Treatment for Metastatic Colorectal Cancer
}

\author{
Min-Sang Lee \\ Samsung Medical Center \\ Yong-Pyo Lee \\ Samsung Medical Center \\ Hongsik Kim \\ Samsung Medical Center \\ Jung Yong Hong \\ Samsung Medical Center \\ Jeeyun Lee \\ Samsung Medical Center \\ Se Hoon Park \\ Samsung Medical Center \\ Joon Oh Park \\ Samsung Medical Center \\ Young Suk Park \\ Samsung Medical Center \\ Ho Yeong Lim \\ Samsung Medical Center \\ Won Ki Kang \\ Samsung Medical Center \\ Seung Tae Kim ( $\sim$ shty1@skku.edu ) \\ Samsung Medical Center
}

\section{Research Article}

Keywords: colorectal cancer, bevacizumab, aflibercept, target therapy

Posted Date: December 23rd, 2020

DOI: https://doi.org/10.21203/rs.3.rs-127099/v1 
License: (c) (i) This work is licensed under a Creative Commons Attribution 4.0 International License. Read Full License 


\section{Abstract}

Background: To date, there are few clinical studies comparing the efficacy and safety of FOLFIRI (folinic acid, fluorouracil and irinotecan) plus bevacizumab or aflibercept in metastatic colorectal cancer patients (mCRC) pretreated with oxaliplatin-based chemotherapy.

Methods: We analyzed the treatment outcomes of patients receiving FOLFIRI in combination with bevacizumab or aflibercept as second-line treatment for mCRC between October 2017 and March 2020. This analysis included 67 patients receiving FOLFIRI plus aflibercept and 83 receiving FOLFIRI plus bevacizumab.

Results: The overall response rate (ORR) was 13.6\% (95\% Cl: 4.85-22.34) in the FOLFIRI-aflibercept group and $14.7 \%$ (95\% Cl: 6.68-22.71) in the FOLFIRI-bevacizumab group. This difference in ORR was not statistically significant. The median progression free survival (PFS) was 8.6 months in the FOLFIRIbevacizumab group and 8.5 months in the FOLFIRI-aflibercept group $(P=0.752)$ (Fig. 1). Patients in the FOLFIRI-bevacizumab group showed a median overall survival (OS) of 12.4 months, while patients in the FOLFIRI-aflibercept group had a median OS of 13.7 months $(P=0.276)$. There were no significant differences in survival between the two treatment groups. The adverse events were also largely similar between the two groups. However, hypertension of grade 3 or more was more frequent in the FOLFIRIaflibercept group.

Conclusion: FOLFIRI plus bevacizumab and FOLFIRI plus aflibercept had similar anti-tumor activities and toxicity profiles when used as second-line therapy in mCRC patients. Based on these data, both aflibercept and bevacizumab are suitable anti-angiogenic agents when used in combination with FOLFIRI for $\mathrm{mCRC}$.

\section{Background}

Colorectal cancer is the second most commonly diagnosed cancer in Korea, with an estimated 25,881 new cases of colorectal cancer being diagnosed in 2017 [1]. It is also the third leading cause of cancerrelated death among both men and women, with an estimated 8,758 people dying of colorectal cancer in 2018 [1]. Treatment for metastatic colorectal cancer (mCRC) commonly involves 5-fluoruracil, leucovorin, and oxaliplatin (FOLFOX) or 5-fluorouracil, leucovorin, and irinotecan (FOLFIRI) as standard first-line treatment [2,3]. More recently, biological therapies targeted against epidermal growth factor (cetuximab and panitumumab), angiogenesis inhibitors (bevacizumab, aflibercept, and ramucirumab), and other therapies (regorafenib, trifluridine/tipiracil) have been added to these combinations [4-7]. The use of targeted agents in first- and second-line treatment can improve both the overall survival and progressionfree survival of mCRC patients [8].

Hurwitz and colleagues reported a significant increase in survival in metastatic colorectal cancer patients when 5-fluoruracil based chemotherapy was combined with bevacizumab [9]. Bevacizumab is a monoclonal antibody targeting the vascular endothelial growth factor (VEGF), thereby preventing its 
angiogenic effects. Bevacizumab is indicated in combination with both first- and second-lines chemotherapies for $\mathrm{mCRC}$ patients [5].

Aflibercept, a recombinant fusion protein consisting of the Fc portion of IgG1 and the binding portions of VEGFR 1 and 2, is an anti-angiogenic agent targeting both VEGFA and PIGF (placental growth factor) [10]. In the VELOUR trial, aflibercept was added to FOLRIFI as second-line therapy in metastatic colorectal cancer patients with disease progression after oxaliplatin-based chemotherapy. When compared to patients treated with placebo-FOLRIFI, patients treated with aflibercept-FOLRIFI had an improved median overall survival (OS) (12.1 vs. 13.5 months, respectively; hazard ratio [HR] 0.82; 95\% Cl, 0.71-0.94) [11]. Progression- free survival (PFS) (6.9 vs. 4.7 months, respectively; HR $0.76 ; 95 \% \mathrm{Cl}, 0.66-0.87$ ), and overall response rate (ORR) $(19.8 \%$ vs. $11.1 \%$, respectively) were also improved in the aflibercept-FOLRIFI group when compared with the placebo-FOLRIFI group [11].

Herein, we analyzed the efficacy and safety of targeted therapies (aflibercept or bevacizumab) used in combination with FOLFIRI as second-line treatment in patients with mCRC.

\section{Methods}

\section{Patients}

From October 2017 to March 2020 at Samsung Medical Center in Seoul, Korea, mCRC patients treated with FOLFIRI-aflibercept $(\mathrm{N}=67)$ or FOLFIRI-bevacizumab $(\mathrm{N}=83)$ after progression on an oxaliplatinbased therapy were analyzed in this study. Patients had measurable disease according to the Response Evaluation Criteria in Solid Tumors (RECIST) 1.1 [12]. The following clinicopathological characteristics were collected for all 150 patients: age, gender, primary site, number of metastatic sites, RAS mutational status, and information on chemotherapy. All patients had pathologically or cytologically proven metastatic or recurrent CRC. The left side of the colon was defined as the descending colon, sigmoid colon, and rectum, while the right side of the colon was defined as the cecum and ascending colon. This retrospective study was approved by the Institutional Review Board of Samsung Medical Center and was conducted in accordance with the ethical principles of the Declaration of Helsinki. And written informed consent was waived because of its retrospective study design.

\section{Chemotherapy}

The choice between of using bevacizumab or aflibercept in combination with FOLFIRI was determined by each patient's physician. In the FOLFIRI-aflibercept group, patients received $4 \mathrm{mg} / \mathrm{kg}$ of aflibercept (intravenously [IV]) over 1 hour on day 1 of cycle 1, followed immediately by the FOLFIRI regimen (irinotecan $180 \mathrm{mg} / \mathrm{m}^{2} \mathrm{IV}$ over 90 minutes with leucovorin $200 \mathrm{mg} / \mathrm{m}^{2} \mathrm{IV}$ over 2 hours, followed by a FU $400 \mathrm{mg} / \mathrm{m}^{2}$ bolus and FU $2400 \mathrm{mg} / \mathrm{m}^{2}$ continuous infusion over 46 hours) on days 1 and 3 of cycle 1 . This treatment regimen was repeated every two weeks until disease progression or death. In the FOLFIRIbevacizumab group, patients received $5 \mathrm{mg} / \mathrm{kg}$ of bevacizumab (intravenously [IV]) over 1 hour on day 1 
of cycle 1, followed immediately by the FOLFIRI regimen, as outlined above. This treatment regimen was repeated every two weeks until disease progression or death.

\section{Statistical analyses}

The statistical analysis of baseline demographics and clinical outcomes was based on all data available up to the cutoff date of March 15,2020. Descriptive statistics were used to compare the patient characteristics. Survival analyses were performed using the Kaplan-Meier method and log-rank tests. Progression-free survival (PFS) was defined as the time from the start of FOLRIFI-aflibercept or FOLRIFIbevacizumab treatment until the date of disease progression or death resulting from any cause. Overall survival (OS) was measured from the start of FOLRIFI-aflibercept or FOLRIFI-bevacizumab treatment to the date of death from any cause. All P-values were two-sided, and statistical significance was set at $\mathrm{P}<$ 0.05. Statistical analysis was performed using the IBM SPSS software package, version 25.

\section{Results}

\section{Patient characteristics}

Baseline patient characteristics are provided in Table 1. The FOLFIRI plus aflibercept treatment was used in 67 patients, while the FOLFIRI plus bevacizumab treatment was applied in 83 patients. Of all 150 patients, 75 (50.0\%) were men and $75(50.0 \%)$ were female. The right side of the colon was the most common location of the primary tumor (62.6\%), and RAS mutations were found in 76 patients $(50.6 \%)$. At the beginning of the second treatment, all patients had ECOG PS $0-1$. The median age was 55.96 years in the FOLFIRI-aflibercept group, and 57.40 years in the FOLFIRI-bevacizumab group. There were no significant differences in the clinical characteristics of patients between the two groups (Table 1). 
Table 1

Baseline characteristics of metastatic colorectal cancer patients

\section{FOLFIRI plus \\ FOLFIRI plus bevacizumab $(\mathrm{N}=83) \quad P$-value \\ aflibercept $(\mathrm{N}=67)$}

Age, years

$\leq 70$ years

$62(92.5 \%)$

$74(89.2 \%)$

$>70$ years

$5(7.5 \%)$

$9(10.8 \%)$

range

$26-73$

$14-80$

$P=0.579$

Gender

male

$36(53.7 \%)$

$39(47.0 \%)$

female

$31(46.3 \%)$

$44(53.0 \%)$

$P=0.511$

First-line treatment

FOLFOX plus bevacizumab

$67(100 \%)$

$71(85.5 \%)$

FOLFOX plus cetuximab

$0(0 \%)$

$12(14.5 \%)$

$P=0.004$

Performance status

$\begin{array}{lll}0 & 18(26.9 \%) & 21(25.6 \%) \\ 1 & 45(67.2 \%) & 59(72.0 \%) \\ 2 & 4(6.0 \%) & 2(2.4 \%)\end{array}$

Site of primary tumor

Rt

$39(58.2 \%)$

$55(66.3 \%)$

$\mathrm{Lt}$

$28(41.8 \%)$

$28(33.7 \%)$

$P=0.396$

Number of metastases

one

$56(83.6 \%)$

$59(71.1 \%)$

two

11 (16.4\%)

21 (25.3\%)

three or more

$0(0.0 \%)$

$3(3.6 \%)$

$P=0.096$

RAS

WT

$23(34.3 \%)$

$40(48.2 \%)$

mutant

35 (52.2\%)

41 (49.4\%)

NA

$9(13.4 \%)$

$2(2.4 \%)$

$P=0.180$

MSI state 


\begin{tabular}{|llll|}
\hline & $\begin{array}{l}\text { FOLFIRI plus } \\
\text { aflibercept }(\mathbf{N}=67)\end{array}$ & FOLFIRI plus bevacizumab (N= 83) & P-value \\
\hline MSS & $52(77.6 \%)$ & $64(77.1 \%)$ & \\
\hline MSI & $1(1.5 \%)$ & $3(3.6 \%)$ & \\
\hline NA & $14(20.3 \%)$ & $16(19.3 \%)$ & \\
\hline Operation & & & \\
\hline absent & $33(49.3 \%)$ & $39(47.0 \%)$ & $P=0.870$ \\
\hline present & $34(50.7 \%)$ & $44(53.0 \%)$ & \\
\hline
\end{tabular}

\section{Treatment outcomes}

The efficacies of FOLFIRI plus aflibercept and FOLFIRI plus bevacizumab are presented in Table 2 . Among the 59 evaluable patients in the FOLFIRI-aflibercept group, one had a complete response (CR) to therapy, seven had a partial response (PR), and forty-two had stable disease (SD), with an overall response rate (ORR) of $13.6 \%(95 \% \mathrm{Cl} 4.85-22.34)$ and a disease control rate (DCR) of $84.8 \%(95 \% \mathrm{Cl}$ 75.63-93.96). Among the 75 evaluable patients in the FOLFIRI-bevacizumab group, three patients had a CR to therapy, eight had a PR, and forty-nine had SD, with an ORR of $14.7 \%(95 \% \mathrm{Cl} 6.68-22.71)$ and a DCR of $80.0 \%$ (95\% Cl 70.94-89.05). There were no significant differences in the ORR and DCR between the two treatment groups.

Table 2

Efficacy of second-line chemotherapy

\begin{tabular}{|llll|}
\hline Response & $\begin{array}{l}\text { FOLFIRI plus } \\
\text { aflibercept }(\mathbf{N}=59)\end{array}$ & $\begin{array}{l}\text { FOLFIRI plus } \\
\text { bevacizumab (N=75) }\end{array}$ & P-value \\
\hline Complete response & $1(1.7 \%)$ & $3(4.0 \%)$ & \\
\hline Partial response & $7(11.9 \%)$ & $8(10.7 \%)$ & \\
\hline Stable disease & $42(71.2 \%)$ & $49(65.3 \%)$ & $P=0.506$ \\
\hline Progressive disease & $9(15.2 \%)$ & $15(20.0 \%)$ & \\
\hline Response rate & $8(13.6 \%)$ & $11(14.7 \%)$ & \\
\hline Disease control rate & $50(84.7 \%)$ & $60(80.0 \%)$ & \\
\hline
\end{tabular}

Survival analyses were done for 134 patients. Among the 150 patients included in this study, 16 patients were excluded from the survival analyses due to loss to follow-up. The median progression-free survival (PFS) was 8.6 months in the FOLFIRI-bevacizumab group and 8.5 months in the FOLFIRI-aflibercept group $(P=0.752)$ (Fig. 1). Patients in the FOLFIRI-bevacizumab group showed a median overall survival 
(OS) of 12.4 months, while patients in the FOLFIRI-aflibercept group had a median OS of 13.7 months ( $P$ $=0.276)$ (Fig. 2). There was no significant difference in survival between the two groups.

\section{Adverse events}

The safety profiles of the treatment regimens are provided in Table 3. Treatment-related adverse events of any grade were reported in $86.5 \%$ of patients treated with FOLFIRI plus aflibercept and in $83.1 \%$ of patients treated with FOLFIRI plus bevacizumab. Adverse events of grade 3 or 4 were reported in $11.9 \%$ of patients in the FOLFIRI-aflibercept group and $2.4 \%$ of patients in the FOLFIRI-bevacizumab group. The most common adverse event was anorexia, followed by nausea, hypertension, neutropenia, and mucositis. There were no significant differences in the incidences adverse events between two regimens.

Table 3

Incidence of adverse events

\begin{tabular}{|c|c|c|c|c|c|}
\hline & $\begin{array}{l}\text { FOLFIRI plu } \\
\text { aflibercept }\end{array}$ & = 67) & $\begin{array}{l}\text { FOLFIRI plu } \\
\text { bevacizum }\end{array}$ & $(\mathrm{N}=83)$ & $P$-value \\
\hline & G1/G2 & G3/G4 & G1/G2 & G3/G4 & \\
\hline & & & & & $P=0.106$ \\
\hline Anorexia & $20(29.8 \%)$ & - & $18(21.7 \%)$ & - & \\
\hline Nausea & $18(26.8 \%)$ & - & $12(14.5 \%)$ & - & \\
\hline Neutropenia & $10(15.0 \%)$ & - & $15(18.1 \%)$ & $1(1.2 \%)$ & \\
\hline General weakness & $8(12.0 \%)$ & $1(1.5 \%)$ & $10(14.5 \%)$ & $1(1.2 \%)$ & \\
\hline Thrombocytopenia & $3(4.5 \%)$ & $1(1.5 \%)$ & $2(2.4 \%)$ & - & \\
\hline Hypertension & $20(29.8 \%)$ & $5(7.5 \%)$ & $10(12.0 \%)$ & - & \\
\hline Diarrhea & 8 (11.9) & - & $10(12.0 \%)$ & - & \\
\hline Asthenia & $5(7.5 \%)$ & - & $8(9.6 \%)$ & - & \\
\hline epistaxis & $2(3.0 \%)$ & $1(1.5 \%)$ & $3(3.6 \%)$ & - & \\
\hline AST/ALT elevation & $2(3.0 \%)$ & - & $2(2.4 \%)$ & - & \\
\hline mucositis & $10(15.0 \%)$ & - & $12(14.4 \%)$ & - & \\
\hline pneumonia & $1(1.5 \%)$ & - & - & - & \\
\hline rash & $1(1.5 \%)$ & - & $1(1.2 \%)$ & - & \\
\hline
\end{tabular}

In the FOLFIRI-aflibercept group, eight patients discontinued treatment due to the adverse event of hypertension. One patient experienced reversible cerebral vasoconstriction syndrome, and fully recovered from it after discontinuation of aflibercept. Another patient experienced a hypertensive crisis after 
aflibercept infusion, and recovered from it after discontinuation of the aflibercept. In the FOLFIRIbevacizumab group, two patients discontinued treatment: one due to the patient's worsening condition and one due to persistent neutropenia.

\section{Discussion}

In the present study, we compared FOLFIRI plus aflibercept with FOLFIRI plus bevacizumab as secondline therapy in $\mathrm{MCRC}$ patients who had experienced disease progression following oxaliplatin-based palliative chemotherapy. Based on this study, we confirmed that FOLFIRI plus bevacizumab and FOLFIRI plus aflibercept had similar anti-tumor activities and toxicity profiles when used as second-line therapy in mCRC patients. Our data support that both aflibercept and bevacizumab are suitable anti-angiogenic agents when used in combination with FOLFIRI for second-line treatment of mCRC.

As described earlier, VELOUR trial reported that adding aflibercept to FOLFIRI for treatment of mCRC patients who experienced disease progression following first-line oxaliplatin-based chemotherapy improved their median OS and PFS when compared to patients treated with FOLFIRI plus placebo group [11]. In other previous study, Alessandro Ottaiano and colleagues conducted a study comparing FOLFIRI plus aflibercept with FOLFIRI plus bevacizumab as second-line therapy in RAS-mutated mCRC patients. Although not statistically significant, the OS showed favorable trend in the FOLFIRI plus aflibercept group (FOLFIRI plus aflibercept: 12.1 months vs. FOLFIRI plus bevacizumab: 8.9 months; $P=0.9331$ ) [13]. In the present study, the median PFS was not significantly different between the FOLFIRI-bevacizumab and FOLFIRI-aflibercept groups (8.61 months vs. 8.55 months, respectively; $P=0.752$ ). Furthermore, the OS was also not statistically different between the two treatment groups (FOLFIRI-bevacizumab: 12.4 months vs. FOLFIRI-aflibercept: 13.7 months; $P=0.276$ ). Therefore, our data were consistent with those of previous studies.

Bevacizumab binds human VEGF-A, but with a markedly lower affinity than aflibercept [14]. The association rate for aflibercept binding to VEGF-A is orders of magnitude faster than that measured for bevacizumab. Similarly, in cell-based bioassays, aflibercept inhibited the activation of VEGFR1 and VEGFR2, as well as VEGF-A-induced calcium mobilization and cell migration, in human endothelial cells more potently than bevacizumab $[10,14,15]$. Binding kinetics and affinity are key determinants of the biological activity of antibody-like drugs. Anti-VEGF therapy has also demonstrated improved results in mCRC when given in combination with chemotherapy $[9,11,16]$. However, not all patients benefit from this type of treatment. Thus, the identification of biomarkers that are able to pinpoint patients who are likely to benefit from anti-angiogenic agents is very important. In previous studies, researchers have tried to find a variety of markers of response to antiangiogenic therapies in patients with colorectal cancer. Osterlund et al. showed that hypertension within the first three months of antiangiogenic treatment was predictive for an improved OS [17]. Circulating levels of PIGF have been shown to increase in response to anti-VEGF treatment. Plasma PIGF levels were highest at the point before progression and patients with high plasma P1GF levels were resistant to treatment with bevacizumab $[18,19]$. Plasma VEGF-A levels were significantly positively associated with worse PFS and OS in $\mathrm{MCRC}$ patients who received either 
FOLFOX with bevacizumab or FOLFIRI with bevacizumab [20]. In other studies of patients treated with regorafenib, a better OS and PFS were observed in patients with lower VEGF-A levels [21]. As described earlier, aflibercept has the propensity to block VEGF-A and -B, as well as placental growth factor (PIGF)-1 and-2, thus preventing their interactions with growth factor receptors $[10,22]$. Switching therapeutic approaches based on the patient's PIGF and VEGF levels to target these alternative mechanisms, through the use of such drugs as aflibercept, may be beneficial. In our study, the efficacy of aflibercept is comparable to that of bevacizumab. Future studies are needed to confirm the importance of serum VEGFA and PIGF levels in terms of using anti-angiogenic agents.

There are limitations to this study. First, it was a retrospective study, and clinically heterogeneous populations are subject to potential biases. Second, the study included a relatively small number of patients with anti-angiogenetic agents making it difficult to draw definite conclusions regarding biomarkers. Third, only Asian patients were analyzed in the study, and differences in genomic profiles and clinical features exist between Western and Eastern patients with solid tumors. Therefore, our findings must be interpreted with a level of caution.

In conclusion, we confirmed that FOLFIRI plus bevacizumab and FOLFIRI plus aflibercept had similar antitumor activities and toxicity profiles when used as second-line therapies in $\mathrm{mCRC}$ patients. Our data support that both aflibercept and bevacizumab are suitable anti-angiogenic agents when used in combination with FOLFIRI.

\section{Abbreviations}

mCRC: metastatic colorectal cancer

FOLFIRI: 5-fluorouracil, leucovorin, and irinotecan

FOLFOX: 5-fluoruracil, leucovorin, and oxaliplatin

VEGF: vascular endothelial growth factor

PIGF: placental growth factor

\section{Declarations}

\section{Ethics approval and consent to participate}

This retrospective study was approved by the Institutional Review Board of Samsung Medical Center and was conducted in accordance with the ethical principles of the Declaration of Helsinki. And written informed consent was waived because of its retrospective study design.

\section{Consent for publication}


Not applicable.

\section{Availability of data and materials}

The datasets used and/or analysed during the current study available from the corresponding author on reasonable request..

\section{Competing interests}

The authors declare that they have no competing interest.

\section{Funding}

None.

\section{Authors' contributions}

MSL, STK and KWK designed the study. JYH, JL, SHP, JOP HYL, WKK and STK performed the experiments. MSL, YPL, HSK and STK analyzed and interpreted the experimental data. YPL, HSK, JYH, JL, SHP and STK provided the discussion and suggestions to the experiments. MSL and STK wrote the manuscript with input from all authors. All authors read and approved the final manuscript.

\section{Acknowledgements}

None.

\section{References}

1. Jung KW, Won YJ, Hong S, Kong HJ, Lee ES. Prediction of Cancer Incidence and Mortality in Korea, 2020. Cancer Res Treat. 2020;52(2):351-8.

2. Zhao Z, Pelletier E, Barber B, Bhosle M, Wang S, Gao S, et al. Patterns of treatment with chemotherapy and monoclonal antibodies for metastatic colorectal cancer in Western Europe. Curr Med Res Opin. 2012;28(2):221-9.

3. Abrams TA, Meyer G, Schrag D, Meyerhardt JA, Moloney J, Fuchs CS. Chemotherapy usage patterns in a US-wide cohort of patients with metastatic colorectal cancer. J Natl Cancer Inst. 2014;106(2):djt371.

4. Das S, Ciombor KK, Haraldsdottir S, Goldberg RM. Promising New Agents for Colorectal Cancer. Curr Treat Options Oncol. 2018;19(6):29.

5. Venook AP, Niedzwiecki D, Lenz HJ, Innocenti F, Fruth B, Meyerhardt JA, et al. Effect of First-Line Chemotherapy Combined With Cetuximab or Bevacizumab on Overall Survival in Patients With KRAS Wild-Type Advanced or Metastatic Colorectal Cancer: A Randomized Clinical Trial. JAMA. 2017;317(23):2392-401. 
6. Chung KY, Shia J, Kemeny NE, Shah M, Schwartz GK, Tse A, et al. Cetuximab shows activity in colorectal cancer patients with tumors that do not express the epidermal growth factor receptor by immunohistochemistry. J Clin Oncol. 2005;23(9):1803-10.

7. Mayer RJ, Van Cutsem E, Falcone A, Yoshino T, Garcia-Carbonero R, Mizunuma N, et al. Randomized trial of TAS-102 for refractory metastatic colorectal cancer. N Engl J Med. 2015;372(20):1909-19.

8. Segelov E, Chan D, Shapiro J, Price TJ, Karapetis CS, Tebbutt NC, et al. The role of biological therapy in metastatic colorectal cancer after first-line treatment: a meta-analysis of randomised trials. $\mathrm{Br} \mathrm{J}$ Cancer. 2014;111(6):1122-31.

9. Hurwitz H, Fehrenbacher L, Novotny W, Cartwright T, Hainsworth J, Heim W, et al. Bevacizumab plus irinotecan, fluorouracil, and leucovorin for metastatic colorectal cancer. New Engl J Med. 2004;350(23):2335-42.

10. Papadopoulos N, Martin J, Ruan Q, Rafique A, Rosconi MP, Shi E, et al. Binding and neutralization of vascular endothelial growth factor (VEGF) and related ligands by VEGF Trap, ranibizumab and bevacizumab. Angiogenesis. 2012;15(2):171-85.

11. Van Cutsem E, Tabernero J, Lakomy R, Prenen H, Prausova J, Macarulla T, et al. Addition of aflibercept to fluorouracil, leucovorin, and irinotecan improves survival in a phase III randomized trial in patients with metastatic colorectal cancer previously treated with an oxaliplatin-based regimen. $\mathrm{J}$ Clin Oncol. 2012;30(28):3499-506.

12. Schwartz LH, Litiere S, de Vries E, Ford R, Gwyther S, Mandrekar S, et al. RECIST 1.1-Update and clarification: From the RECIST committee. Eur J Cancer. 2016;62:132-7.

13. Ottaiano A, Capozzi M, Tafuto S, De Stefano A, De Divitiis C, Romano C, et al. Folfiri-Aflibercept vs. Folfiri-Bevacizumab as Second Line Treatment of RAS Mutated Metastatic Colorectal Cancer in Real Practice. Front Oncol. 2019;9:766.

14. Yu L, Liang XH, Ferrara N. Comparing protein VEGF inhibitors: In vitro biological studies. Biochem Biophys Res Commun. 2011;408(2):276-81.

15. Jocelyn Holash SD, Nick Papadopoulos, Susan D. Croll, Lillian Ho, Michelle Russell, Patricia Boland, Ray Leidich,, Donna Hylton EB, Ella loffe, Tammy Huang, Czeslaw Radziejewski, Kevin Bailey, James P. Fandl, Tom Daly, Stanley J. Wiegand GDY, and John S. Rudge. VEGF-Trap: A VEGF blocker with potentantitumor effects. PNAS. 2002;99:11393-8.

16. Sun W. Angiogenesis in metastatic colorectal cancer and the benefits of targeted therapy. J Hematol Oncol. 2012;5:63.

17. Osterlund P, Soveri LM, Isoniemi H, Poussa T, Alanko T, Bono P. Hypertension and overall survival in metastatic colorectal cancer patients treated with bevacizumab-containing chemotherapy. $\mathrm{Br} \mathrm{J}$ Cancer. 2011;104(4):599-604.

18. Kopetz S, Hoff PM, Morris JS, Wolff RA, Eng C, Glover KY, et al. Phase II trial of infusional fluorouracil, irinotecan, and bevacizumab for metastatic colorectal cancer: efficacy and circulating angiogenic biomarkers associated with therapeutic resistance. J Clin Oncol. 2010;28(3):453-9. 
19. Van Cutsem E, Paccard C, Chiron M, Tabernero J. Impact of Prior Bevacizumab Treatment on VEGF-A and PIGF Levels and Outcome Following Second-Line Aflibercept Treatment: Biomarker Post Hoc Analysis of the VELOUR Trial. Clin Cancer Res. 2020;26(3):717-25.

20. Parikh AR, Lee FC, Yau L, Koh H, Knost J, Mitchell EP, et al. MAVERICC, a Randomized, Biomarkerstratified, Phase II Study of mFOLFOX6-Bevacizumab versus FOLFIRI-Bevacizumab as First-line Chemotherapy in Metastatic Colorectal Cancer. Clin Cancer Res. 2019;25(10):2988-95.

21. Suenaga M, Mashima T, Kawata N, Wakatsuki T, Horiike Y, Matsusaka S, et al. Serum VEGF-A and CCL5 levels as candidate biomarkers for efficacy and toxicity of regorafenib in patients with metastatic colorectal cancer. Oncotarget. 2016;7(23):34811-23.

22. Van de Veire S, Stalmans I, Heindryckx F, Oura H, Tijeras-Raballand A, Schmidt T, et al. Further pharmacological and genetic evidence for the efficacy of PIGF inhibition in cancer and eye disease. Cell. 2010;141(1):178-90.

\section{Figures}

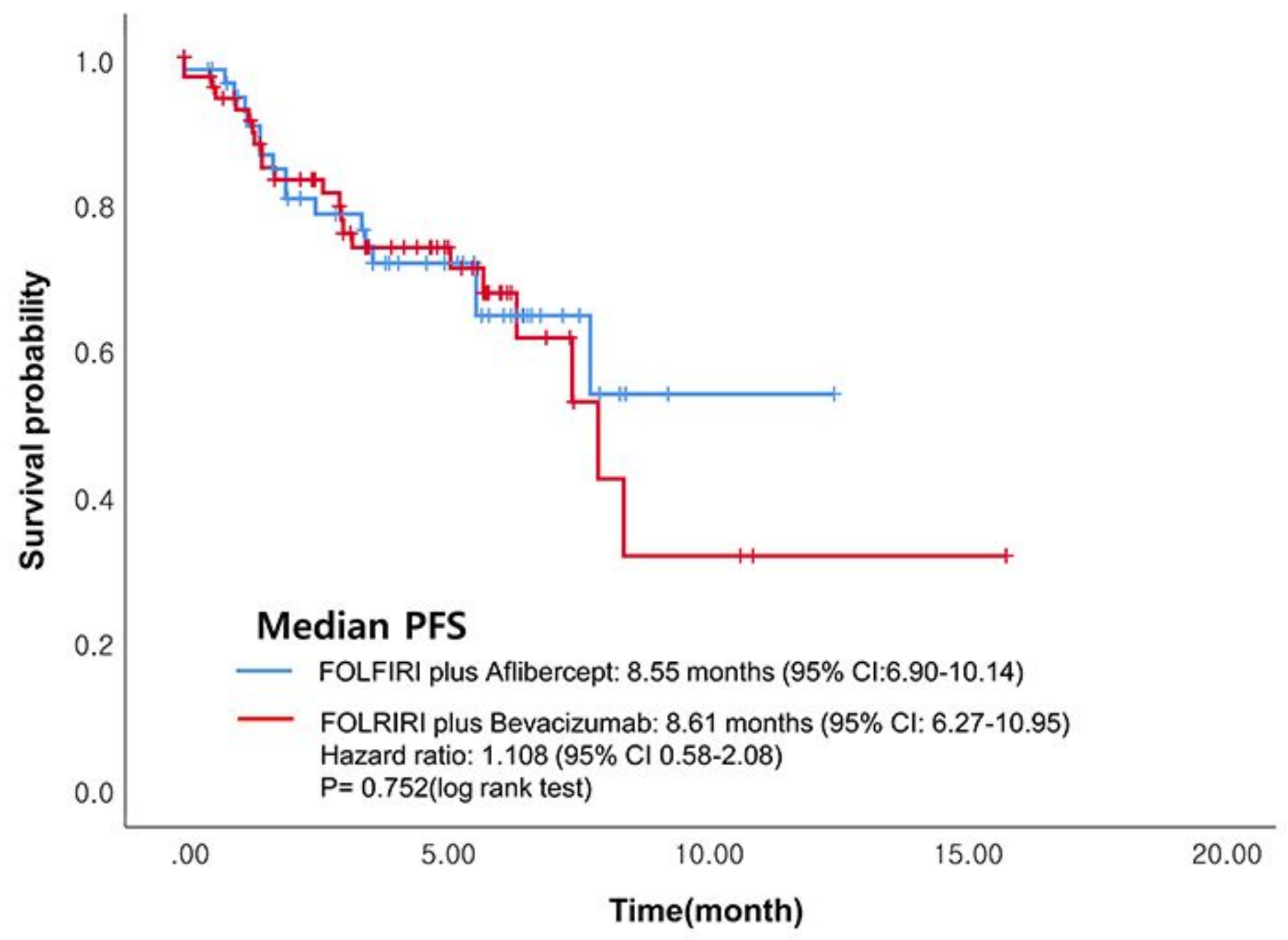

Figure 1 
Kaplan-Meier curves for progression-free survival (PFS). FOLFIRI plus aflibercept group median PFS was 8.55 months (95\% $\mathrm{Cl}$ 6.9-10.14) and FOLFIRI plus bevacizumab group median. PFS was 8.61 months (95\% Cl:6.27-10.95).

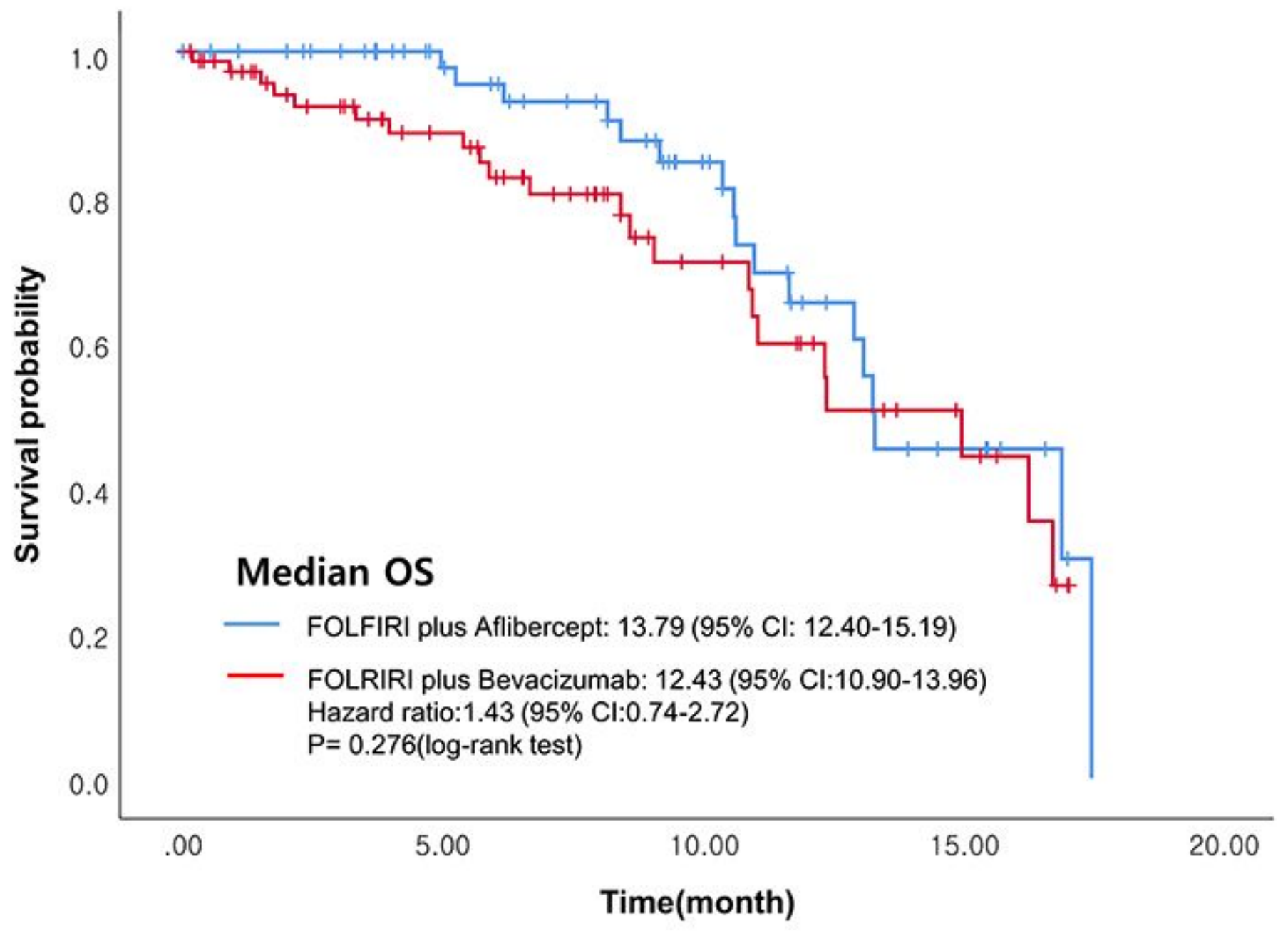

Figure 2

Kaplan-Meier curves for overall survival. FOLFIRI plus aflibercept group median OS was 13.79 months (95\% Cl:12.40-15.19) and FOLFIRI plus bevacizumab group median OS was 12.43 months (95\% Cl:10.9013.96). 